\title{
HEART RATE RESPONSES DURING TRANSFERRING A PARAPLEGIC PATIENT AT DIFFERENT HEIGHTS
}

\author{
Kwan-Hwa Lin ${ }^{1}$, Tung-Wu Lu², Ssu-Yuan Chen ${ }^{3}$ \\ ${ }^{1}$ Graduate Institute and School of Physical Therapy, College of Medicine, \\ National Taiwan University, \\ ${ }^{2}$ Institute of Biomedical Engineering, College of Medicine, National Taiwan University, \\ ${ }^{3}$ Department of Physical Medicine and Rehabilitation, National Taiwan University Hospital, \\ Taipei, Taiwan
}

\begin{abstract}
Transferring a patient is a basic assistance for individuals with moderate to maximum disability. The purpose of this study was to investigate the heart rate (HR) and workload of physical therapists during transferring a paraplegic patient from a newly designed wheelchair with adjustable height. Eleven healthy therapists $(24 \pm 1$ years of age, $n=11)$ transferred the same thoracic cord (T12) injured male (weight $52 \mathrm{~kg}$ ) from the wheelchair to bed at different height. The HR responses of the therapists were recorded by a telemetry system (WEP-4204J, Nihon Kohden Corp., Tokyo, Japan) to calculate the relative workload ((HRpeak-HRrest)/(HRmax-HRrest)*100\%). One-way ANOVA and Bonferroni test were used to compare the differences among different transfer conditions. There was no significant difference among peak HR during low-even (115.3 \pm 4.6 beats/min), uneven $(130.6 \pm$ 5.3 beats $/ \mathrm{min})$, and high-even transfer (121.9 \pm 5.5 beats/min). The relative workload $(42.2 \pm 3.9 \%)$ during uneven transfer was significantly $(p<0.05)$ higher than that $(28.4 \pm 2.9 \%)$ during low-even transfer, and the difference was abolished during high-even transfer. The conclusion was that the workload of the therapists during uneven transfer was significantly increased and it could be reduced with the modification of the height of the wheelchair or bed.
\end{abstract}

Biomed Eng Appl Basis Comm, 2006(April); 18: 95-99.

Key words: transfer, heart rate, workload, paraplegia

\section{INTRODUCTION}

Transferring a patient is a basic functional assistance for individuals with moderate to maximum disability [1-4]. The one who performs the transfer includes the health professionals, and the attendant

Received: Nov. 11, 2005; Accepted: Feb. 17, 2006

Correspondence: Tung-Wu Lu, Professor

Institute of Biomedical Engineering, National Taiwan University, \#1, Sec. 1, Jen-Ai Road, Taipei 100, Taiwan

E-mail: twlu@ntu.edu.tw caregivers [5-7]. The health professionals, especially nurses and physical therapists, have been identified as the high risk group with low-back injury [8-9]. One of the possible reasons for low-back injury is suggested as the high physiological work demand during transfer [10-11]. The workload on heart with continuous heart rate (HR) monitor in nurses demonstrated that the HR during solo transfer was $129 \pm 10.9$ beats $/ \mathrm{min}$, and the rate of perceived exertion was $15 \pm 3.2$ which indicated heavy workload [11]. However, there is lack of quantitative analysis of workload on therapists during 
transferring the disabled patients at different heights. Transferring patients can be performed by manual techniques and with different mechanical aids [7]. The mechanical aids, such as: Hoyer lift, and Trans-aid were not perceived by nurses and patients to be comfortable and less physical stressful [7]. Therefore, manual transfer is still the major one for delivering the patient. Transfer is a brief, heavy workload [10] and it took about 20 seconds to transfer patients from wheelchair to shower chair [7]. Therefore, it is difficult to measure the steady-state oxygen consumption (i.e., $>3 \mathrm{~min}$ ) as the intensity of workload. The heart rate measurement would be used in this study to calculate the workload [11-12]. Furthermore, the physical therapists and physical therapy students were recruited as the one who performed the patient transferring task.

From the usage survey of ambulatory assistive devices in long-term care facilities in Taiwan, the height of regular wheelchair is $50.2 \pm 10.2 \mathrm{~cm}$ $(\mathrm{n}=200)$, the bed is $51.2 \pm 10.6 \mathrm{~cm}(\mathrm{n}=213)$ and the regular wheelchair is $49.4 \pm 10.9 \mathrm{~cm}(\mathrm{n}=192)$ [13]. If the bed mattress or seat cushion is added, the total height of bed or wheelchair would be around $60 \mathrm{~cm}$. Therefore, the uneven transfer is common for patient care. We have designed a new wheelchair with adjusted height (ROC patent number 181375). The research hypotheses of this study included: (1) uneven transfer would increase the heart rate and workload, and (2) the increase of the heart rate and workload could be overcome by adjusting height of wheelchair or bed.

\section{MATERIAL AND METHOD}

\section{Subjects}

Eleven healthy adults who performed the patient transfer included six physical therapists (PT) with one to seven years of working experience, and five physical therapy students (PTS) with clinical training and experience of patient transfer. In order to keep the workload quite constant, all the therapists transferred the same patient and the study finished within 2 weeks.

According to the classification of American Spinal Injury Association (ASIA) [14], the patient who was transferred by the therapists was a thoracic cord motor complete injured male with neurological level at T12, and the ASIA impairment scale was B (complete motor loss below the level of injury with sensory preservation). He was 21 years old with traffic accident happened 3 months ago. His body weight was $52 \mathrm{~kg}$ and height was $170 \mathrm{~cm}$. During the time of study, he could perform the transfer by himself with minimum assistance. However, the patient was instructed not to use his feet on the floor to aide the transfer, and had to be a passive patient. The consent form was obtained and this study has been proved by the Institutional Review Committee of the National Taiwan University Hospital, Taipei, Taiwan.

\section{Set-up and Protocol}

The adjustable wheelchair with maximum elevation of $18 \mathrm{~cm}$ height and maximal lateral-shift of $12 \mathrm{~cm}$ distance (Fig. 1A and B) was designed by our research group and was made in Taipei, Taiwan. The newly designed adjustable wheelchair was put along the side of the bed with adjustable height, and the approaching angle was 30 degree. The patient sat on the wheelchair, and the therapist stood in front of the patient with his/her hands holding patient's waist and belt. All the therapists transferred the same patient twice for each of the following protocols in a random order: (1) Low-even transfer: the wheelchair was at the same height as the bed (i.e., $58 \mathrm{~cm}$ height from floor). (2) Uneven transfer: the bed was 8 higher than the wheelchair. (3) High-even transfer: the wheelchair was elevated and lateral shifted to match the raised bed (i.e., $66 \mathrm{~cm}$ height from floor).

\section{Heart Rate Measurement and Relative Workload}

The heart rate was measured by a telemetry system (WEP-4204J, Nihon Kohden Corp., Tokyo, Japan). The surface EKG electrodes were put on the right $2^{\text {nd }}$ intercostal space and the left $5^{\text {th }}$ intercostal space.

The resting heart rate $\left(\mathrm{HR}_{\text {rest }}\right)$ was measured before the trial and about 10 minutes rest was monitored between each trial. The peak heart rate $\left(\mathrm{HR}_{\text {peak }}\right)$ was recorded for each trail. The relative workload was calculated by the formula of Rodah (1989) as $\left(\mathrm{HR}_{\text {peak }}-\mathrm{HR}_{\mathrm{rest}}\right) /\left(\mathrm{HR}_{\max }-\mathrm{HR}_{\text {rest }}\right) \times 100 \%$.
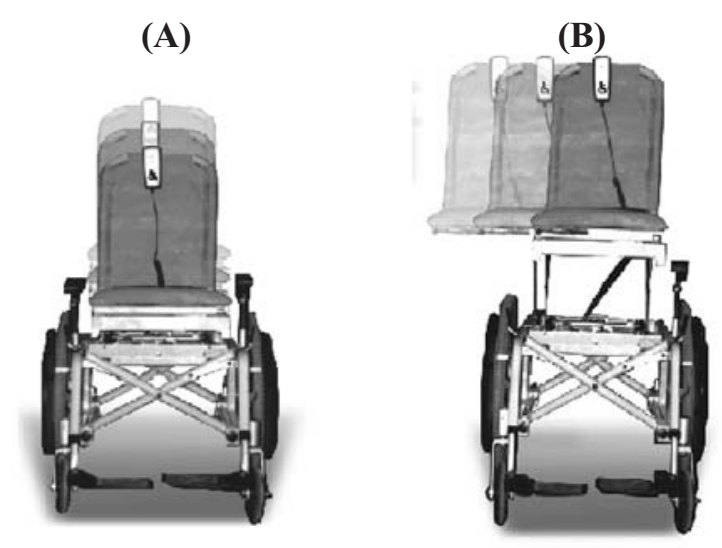

Fig 1. A newly designed wheelchair with elevation (A) and lateral shift (B) functions. 
The maximum HR $\left(\mathrm{HR}_{\max }\right)$ was determined from 220 minus age [12].

\section{Data Analysis}

All the data was stored in SPSS 11.0 for Window. The mean and standard error (SE) was calculated by the descriptive statistics. The mean $\mathrm{HR}_{\text {peak }}$ was calculated as the mean of the two trials. The comparison of workload among three conditions was analyzed by one-way ANOVA and Bonferroni posthoc analysis. The statistical significance was set at $\mathrm{p}<0.05$.

\section{RESULT}

The mean age of the 11 therapists (including PTS) was $24.4 \pm 1.1$ years old, and the mean body weight and height were $62.7 \pm 3.5 \mathrm{~kg}$, and $166.4 \pm 2.4 \mathrm{~cm}$, respectively (Table I ). The age of PTS was significantly younger than PT (Table I ).

The mean resting HR is $83.9 \pm 3.1$ beats/min, and the peak HR during even or uneven transfer was significantly $(p<0.05)$ greater than that at rest. However, there was no significant difference of HRpeak among low-even $(115.3 \pm 4.6$ beats $/ \mathrm{min})$, uneven (130.6 \pm 5.3 beats/min)and high-even $(121.9 \pm$ 5.5 beats/min) transfer (Fig. 2).
In terms of relative workload, the uneven transfer $(42.1 \pm 3.9 \%)$ significantly $(\mathrm{p}=0.045)$ increases the workload more than that during low-even transfer $(28.4 \pm 2.9 \%)$, and the increase is abolished during high-even transfer $(34.0 \pm 4.2 \%)$ with elevated, lateral-shift wheelchair (Fig.3).

\section{DISCUSSION}

This study indicated that the even and uneven transfer significantly increased the heart rate as comparing with the resting state (HRrest=83.9 \pm 4.6 beats $/ \mathrm{min}$ ). Therefore, transferring a patient is a demanding task. The mean heart rate responses during low-even and uneven transfer in this study were 115.3 \pm 4.6 and $130.6 \pm 5.3$ beats $/ \mathrm{min}$, which were close to the report from Hui-Ling et al. whom measured the heart rate responses of nurses doing the transferring task in the geriatric ward (i.e., $129 \pm 10.9$ beats $/ \mathrm{min}$ ) [11]. It seemed that the HR response did not differ much between nurses and physical therapists, although we did not measure the transferring heart rate responses both in nurses and physical therapists.

The workload can be measured from oxygen consumption [15], heart rate [12], and systematic blood pressure [16]. The transient work (i.e., few seconds) during transfer is difficult to obtain from the

Table I . Demographic data of participants.

\begin{tabular}{cccccc}
\hline NO. & Type & Age (Y.O.) & Sex & Weight $(\mathrm{Kg})$ & Height $(\mathrm{cm})$ \\
\hline 1 & PTS & 21 & M & 57 & 168 \\
2 & PTS & 21 & $\mathrm{~F}$ & 56 & 160 \\
3 & PTS & 22 & $\mathrm{~F}$ & 50 & 155 \\
4 & PTS & 22 & $\mathrm{~F}$ & 51 & 159 \\
5 & PTS & 22 & $\mathrm{M}$ & 70 & 174 \\
\hline Mean & & $21.6^{*}$ & & 56.8 & 163.2 \\
SE & 1.3 & & 3.6 & 3.4 \\
\hline 6 & PT & 23 & $\mathrm{~F}$ & 55 & 162 \\
7 & PT & 25 & $\mathrm{~F}$ & 50 & 158 \\
8 & PT & 25 & $\mathrm{M}$ & 80 & 178 \\
9 & PT & 26 & $\mathrm{M}$ & 78 & 174 \\
10 & PT & 30 & $\mathrm{M}$ & 74 & 174 \\
11 & PT & 31 & $\mathrm{M}$ & 69 & 168 \\
\hline Mean & 26.7 & & 67.7 & 169.0 \\
SE & 1.3 & & 5.1 & 3.2 \\
\hline Total Mean & 24.4 & & 62.7 & 166.4 \\
SE & 1.1 & & 3.5 & 2.4 \\
\hline PTS: physical therapy student & & & \\
PT: physical therapist & & & & \\
M: male & & & & \\
F: female & & & & \\
$*$ : $<$ 0.05, if PTS v.s PT &
\end{tabular}




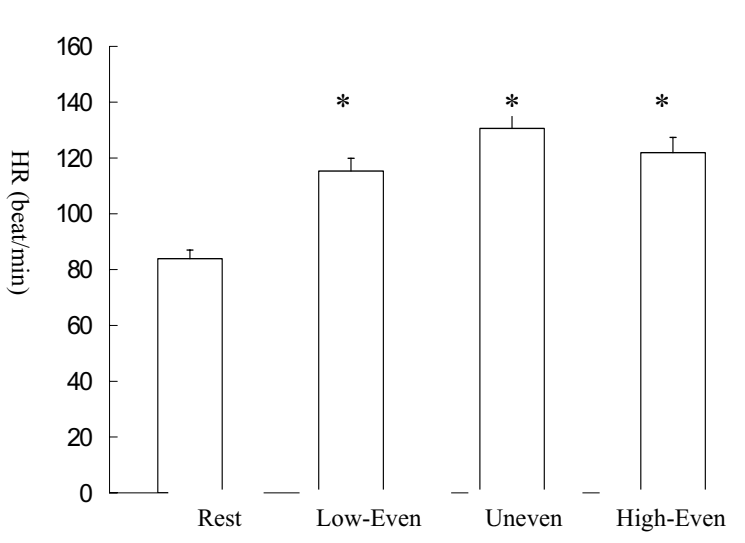

Fig 2. The peak heart rate (HRpeak) responses during rest, low-even transfer, uneven transfer, and high-even transfer. $* \mathrm{p}<0.05$, if compared with resting HR.

measurement of oxygen consumption and blood pressure. The measurement of recovery heart rate responses has been proposed to calculate the workload of static combined with dynamic task [17]. However, it was difficult to measure the recovery heart rate if the therapists had to readjust her posture after transfer. The recovery heart rate was not measured in this study. In stead, the relative heart rate increase during transfer was considered as a reliable indicator of workload [12]. The relative workload in this study is calculated from the formula of Rodahl (1989), and it is related to heart rate reserve (HRmax-HRrest) [12].

This study indicated that when transferred a subject from low to high level (i.e., uneven transfer) significantly increased the relative heart rate as comparing with low-level transfer, but the peak heart rate did not increase significantly. Therefore, the uneven transfer increased the relative load on heart, not the absolute heart rate response; in order to lift the patient against gravity. The result supported part of our hypothesis that the uneven transfer from low to high level increased the relative physical demand of the heart. As shown in Figure 3, this study also supported that the relative work demand on heart during uneven transfer could be overcome by the elevated and lateralshift wheelchair (i.e., high-even transfer).

The workload difference during even and uneven transfer might be explained by the differences caused by the gravity effect and the use of body mechanics. During uneven transfer, therapist would have to lift up the patient to against the gravity, and the target level might be too high for the therapist to use their knees to hold the patient knees for pivoting transfer with body mechanics. This probably is the reason to increase the workload during uneven transfer. During high-even

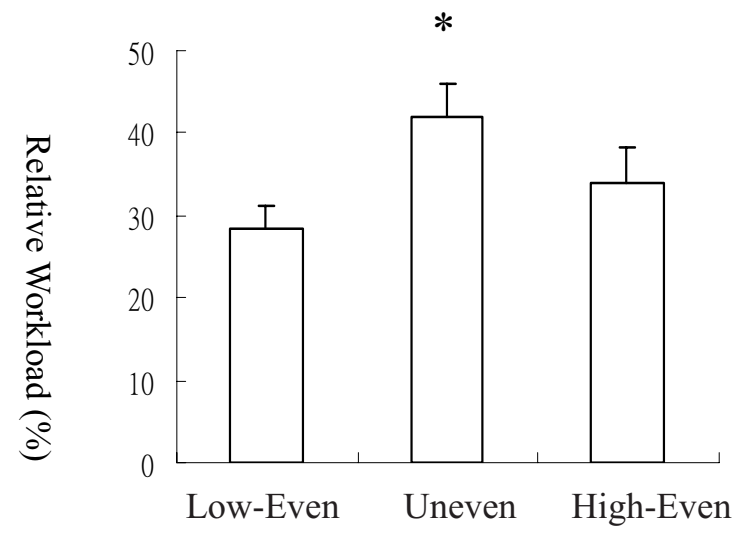

Fig 3. The relative workload during patient transfer $(\mathbf{n}=11){ }^{*}: \mathrm{p}<0.05$, if compared with low-even transfer.

transfer, the therapist did not have to lift the patient up, but the therapist had to pull the patient laterally by upper extremity and could not rely on the lower extremity pivoting force. Therefore, the workload was reduced, but the peak heart rate response did not significantly different from that during uneven transfer.

In spite of the height of transfer, the possible factors influencing the transfer included skill, sex, anthropometry, and workplace (Ryden 1989, Garg 1991). If compared the peak heart rate responses between experienced physical therapists and nonexperienced students, there was no significant difference found. The limitation of this study was that we did not control the sex and anthropometry of the therapists. However, the strength of this study is that the workplace and the subject being transferred were controlled. Further studies would be suggested to investigate the factors influencing the transfer.

The major conclusion of this study is that the workload during uneven transfer would be increased and the adjustable seat height would reduce the workload. However, the strain on the low back would require further investigation.

\section{ACKNOWLEGEMENT}

The authors thank Mr. Ching-Mu Shue who helped the design and manufacture of the wheelchair. This project was sponsored by a grant from National Science Council, Taipei, Taiwan (NSC 91-2614-B-002001-M47). 


\section{REFERENCE}

1. Disler PB, Roy CW, Smith BP: Predicting hourse of care needed. Arch Phys Med Rehabil 1993; 74:139-143.

2. Saboe LA, Darrah JM, Pain KS, et al: Early predictors of functional independence 2 years after spinal cord injury. Arch Phys Med Rehabil 1997; 78:644-650.

3. Samsa GP, Hoening H, Branch LG.: Relationship between self-reported disability and caregiver hours. Am J Phys Med Rehabil 2001; 80:674-684.

4. Tooth L, McKenna K, Geraghty T: Rehabiiltation outcomes in traumatic spinal cord injury in Australia: functional status, length of stay and discharge setting. Spinal Cord 2003; 41:220-230.

5. Klein BP, Jensen RC, Sanderson LM: Assessment of workers compensation claims for back strains/sprains. J Occup Med 1984; 26:443-448.

6. Schleichkorn J, Jagerman F: The need for safety and accident prevention in the clinic. Clinical Management 1986; 6:22-29.

7. Garg A: A biomechanical and ergonomic evaluation of patient transferring tasks: wheelchair to shower chain and shower chain to wheelchair. Ergonomics 1991; 34:407-419.

8. Molumphy M, Unget B, Jensen G, et al: Incidence of work-related low back pain in physical therapists. Phys Ther 1982; 65:482-486.

9. Ryden LA, Molgaard CA, Bobbitt S, et al: Occupational low-back injury in a hospital employee population: an epidemiologic analysis of multiple risk factors of a high-risk occupational group. Spine 1989; 14:315-320.

10. Josephson M, Hagberg M, Hjelm EW: Selfreported physical exertion in geriatric care: a risk indicator for low back symptoms. Spine 1996; 21:2781-2785.

11. Hui L, Ng GYF, Yeung SSM, et al: Evaluation of physiological work demands and low back neuromuscular fatigue on nurses working in geriatric wards. Appl Ergon 2001; 32:479-483.

12. Rodahl K. The physiology of work. Taylor and Francis, London. 1989.

13. Hu MH, Rung YM: A usage survey and user training project of locomotion assistive devices. The Report of Research Project (NSC 90-2614-B002-005-M47) sponsored by National Science Council, Republic of China, 2001.

14. Maynard FM, Bracken MB, Creasey G, et al: International standards for neurological and functional classification of spinal cord injury. Spinal Cord 1997; 35:266-274.

15. Astrand PO, Rodahl K: Textbook of work physiology. New York: McGraw-Hill, 1986.
16. Asfour SS, Genaidy AM, Khalil TM, et al: Physiologic response to static, dynamic and combined work. Am Indus Hygiene Asso J 1986;47:798-802.

17. Chen Y-L, Lee YH: Effect of combined dynamic and static workload on heart recovery cost. Ergonomics 1998;41:29-38. 